\title{
„Manipulation” und kein Ende? - Ein Plädoyer für die Entwicklung einer kommunikationspolitischen Gesamtkonzeption
}

\author{
von Joachim Westerbarkey
}

1. Die Diskussion um die Wirkungen der Massenmedien ${ }^{1}$ ist bekanntlich genau so alt wie die Massenmedien selber. Politiker und Juristen, Lehrer und Theologen, Dichter und Denker und seit mehr als einem halben Jahrhundert auch Soziologen, Psychologen und endlich Zeitungs-, Publizistik- und Kommunikationswissenschaftler: sie alle und alle Welt ergehen sich unermüdlich in allerlei Mutmaßungen über das Phänomen "Wirkung“. Spätestens nach der Erfindung des Buchdrucks, als offensichtlich wurde, daß derartige neue technische Errungenschaften höchst brisante politische, kulturelle und ideologische Folgen haben können, begann ein vielstimmiges Konzert von Hoffnungen, Befürchtungen, Mahnungen, Würdigungen und Verteufelungen - vom Pro der informationsfreundlichen Aufklärung bis zum Contra der Kulturpessimisten. Die meisten Diskutanten beurteilen noch heute die mutmaßlichen Nutzen oder Gefahren der Massenmedien vornehmlich nach ihren eigenen politischen bzw. ideologischen Interessen, messen also den Wert der Medien am Normenkodex ihrer Weltanschauung. Dabei geht es so gut wie nie um das Problem der publizistischen Wirkungen selbst, sondern fast immer um erwünschte oder mißliebige Richtungen und Qualitäten von Inhalten massenmedialer Kommunikation, von denen irrtümlich bruchlos auf Wirkungen geschlossen wird.

Vertreter von Interessengruppen jedweder Prägung schätzen gewöhnlich den Einsatz von Massenmedien zu eigenen $Z$ wecken und betrachten die Beeinflussung von Menschen mittels Medien nicht nur als ihr gutes Recht, sondern als ihre weltanschauliche Pflicht. Kritisiert werden daher in der Regel nur konkurrierende Inhalte, d. h. selten die Massenmedien selbst bzw. deren unterstelltes Wirkungspotential an sich, auf das heute schließlich alle politischen Strategen, religiösen Verkünder und kommerziellen Werber rechnen müssen. ${ }^{2}$

2. Spätestens seit der Blüte der "kritischen Theorie“ in der BRD der 60er Jahre und in verstärktem Maße seit der hiesigen Renaissance des Marxismus wenig später in nahezu allen Sozialwissenschaften wird nun nicht mehr allein von guten und bösen Wirkungen, sondern auch allenthalben von der "Manipulation“ der Menschen durch die Massenmedien gesprochen. Mit dieser zumeist eindeutig pejorativ gemeinten Behauptung ist die bedrückende Vision der Meinungsmarionette Einzelmensch an den Fäden einiger übermächtiger publizistischer Institutionen verbunden, die unter der Sammelbezeichnung "Bewußtseinsindustrie“ ihrerseits als Werkzeuge bzw. Agenturen „der Herrschenden " eingeordnet werden, womit vor allem die großen Kapitalund Produktionsmittelbesitzer und deren Spitzenfunktionäre im Wirtschaftssystem angesprochen sind, aber auch die Partei- und Kirchenführer und die übrigen Vertreter mächtiger Interessengruppen. Sie allesamt, so wird argumentiert, vertreten direkt oder indirekt die Interessen des Monopol- oder Großkapitals, weil sie z. B. jene spätbürgerlich-liberale Konkurrenz- und Konsumideologie auf der Basis privater Eigentumsverhältnisse befürworten oder wenigstens nicht entschieden kritisieren. Die demo-

Dr. Joachim Westerbarkey ist Akademischer Oberrat am Institut für Publizistik der Universität Münster/Westf. 
kratische Verfassung, heißt es, werde dabei auf ihren formalen Anspruch reduziert, auf die "Oberflächenstruktur " der Gesellschaft, unterhalb derer das "Establishment “ ungestört seinen undemokratischen Zielen nachgehen könne. $\mathrm{Da}$ nun gleichzeitig klar wurde, daß das Heer der Lohnabhängigen zur Zeit wenig Wert auf revolutionäres Bewußtsein legt und das Wort „Proletarier" nach wie vor für ein Schimpfwort hält, besannen sich die Kritiker auf Schnädelbachs Satz von der Ideologie als "gesellschaftlich notwendig falschem Bewußtsein “. Zu den Spitzenagenten dieses "falschen Bewußtseins" und seiner Produktion werden neben den Verantwortlichen in den Massenmedien die Vertreter aller anderen Erziehungs- und Bildungseinrichtungen wie z. B. der Familien und Schulen gerechnet, die zum gesellschaftlichen "Überbau" zählen und als durch die historische Entwicklung mehr oder weniger von der ökonomischen Basis „entfremdet" abqualifiziert werden. Wenn dabei von Manipulation die Rede ist, werden im großen und ganzen nicht einmal böse Absichten unterstellt; denn Manipulation wird als notwendige Folge derartiger struktureller Widersprüche in der Gesellschaft betrachtet.

3. Mit Gross kann man allerdings auch davon ausgehen, daß es seit eh und je in "der Offentlichkeit ${ }^{\text {" }}$ konkurrierende Manipulationsversuche gibt, daß eine manipulationsfreie Gesellschaft womöglich gar nicht $\mathrm{zu}$ verwirklichen ist, auch wenn vor allem in primär marktwirtschaftlich orientierten Sozialsystemen dem Streben ökonomischer Machtgruppen nach „Manipulationsmonopolen“ Vorschub geleistet wird. "Offentlichkeit" ist hier also stets und mit wechselndem Erfolg zugleich Feld und Objekt von Manipulationen seitens dieser oder jener Interessenten. Gesellschaft ohne konkurrierende Gruppen, ohne Werbung, Propaganda und Agitation gab es in der Tat bisher nicht; und solange es solchen gesellschaftlichen Wettbewerb gibt, wird wohl stets zu beobachten sein, daß konkurrierende Gruppen sich gegenseitig Manipulation vorwerfen, für sich selbst aber Information, Bildung und Aufklärung im Sinne redlicher Ziele in Anspruch nehmen. Die Geister scheiden sich aber nicht nur am schwer nachprüfbaren Kriterium der Wahrheit und am Ethos der Aufrichtigkeit, sondern auch am Katalog erlaubter und unerlaubter Mittel, an den "Spielregeln“; denn es wird gewöhnlich auch ein direkter und ungebrochener Wirkungszusammenhang zwischen der Verwendung und Rezeption publizistischer Techniken unterstellt, und $z$ war ebenso unreflektiert und ungeprüft wie bei den Inhalten der Massenkommunikation.

4. Bezieht man den Manipulationsbegriff allerdings allein auf die technische und inhaltliche Verarbeitung von Informationsmaterial, dann wird er zur Selbstverständlichkeit. Eine Veröffentlichung von in diesem Sinne "unmanipulierten " Informationen erweist sich nämlich deshalb als unmöglich, weil die professionelle journalistische Arbeit wie überhaupt jeder Kommunikationsprozeß auf dem $Z$ wang zur dauernden Auswahl und Gestaltung beruht, dem jeder Beteiligte unterworfen ist. Enzensberger stellt logisch fest, daß jeder Umgang mit technischen Medien auf der

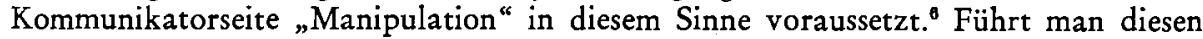
Gedanken in diesem Sprachgebrauch konsequent weiter, dann ist jeder Kommunikator stets Manipulator. Deshalb muß ernsthaft gefragt werden, ob ein umgangssprachlich so negativ besetzter Begriff wie "Manipulation“ noch sinnvoll zu gebrauchen ist, wenn er nichts weiter als das Kommunikationsprinzip selbst meint? Durch seine Verallgemeinerung auf alles ${ }_{n}$ zielbewußte technische Eingreifen in ein gegebenes Material “ (Enzensberger) wird er eigentlich überflüssig, weil es für diesen Vorgang bereits jene Kategorien "Auswahl" und "Gestaltung" gibt, die sich wissenschaftlich bewährt haben. 
5. Angesichts solcher Ärgernisse mit der Brauchbarkeit eines Begriffs, der doch in aller Munde ist, aber von den einen als totaler Wirkungszusammenhang herrschender gesellschaftlicher Strukturen und Ideologien verstanden, von den anderen als unlautere Praktiken des jeweiligen Interessenkonkurrenten qualifiziert und von den dritten auf jeden Umgang mit technischen Medien bezogen wird; angesichts dieser Ärgernisse gilt es dringend, klärende und nachprüfbare Abgrenzungen vorzunehmen, die notwendig normativ erfolgen müssen, weil der Manipulationsbegriff schon umgangssprachlich ein Politikum bezeichnet, das nicht durch eine Beschränkung auf Kriterien technischer Operation aus der Welt geschafft werden kann. „Manipulation“ im üblichen Verständnis verweist nämlich immer auf die Wertfrage: auf Kommunikationsethik, auf soziale Normen, auf das Problem abweichenden Verhaltens etc. Um einmal $\mathrm{zu}$ demonstrieren, wie einem solchen Politikum begrifflich-operational Rechnung getragen werden könnte, nehme ich deshalb eine Arbeitsdefinition, die auf eine Reihe von Überprüfungsmöglichkeiten hinweist, zum Anlaß, das Phänomen selbst zu diskutieren:

„Der (negative) Tatbestand der Manipulation gilt als erfüllt, wenn unter Nichtaufdeckung der eigenen bzw. unter Vorgabe anderer Interessen bzw. Ziele und wissentlich zum Schaden der Betroffenen Informationen unterschlagen oder verfälscht werden." Und als Zusatz: "Selektion von Information kann, muß aber in diesem Sinne nicht Manipulation sein. ${ }^{\text {"7 }}$

An dieser Konstruktion fällt zunächst (genau wie an der von Enzensberger) auf, daß sie primär auf das publizistische Handeln des Kommunikators abhebt und den Rezipienten sowie das Problem publizistischer Wirkungen nur indirekt berücksichtigt. Auf diesen Mangel wird später zwar noch eingegangen, doch erklärt er sich vor allem aus den Schwierigkeiten einer exakten Überprüfung von Wirkungen der Massenkommunikation. $^{8}$

6. Doch auch eine Überprüfung des oben definierten "Tatbestandes" fällt schwerer, als zunächst erwartet. Während die Nichtaufdeckung eigener Interessen in der Nachrichtenpraxis gang und gäbe ist und im journalistischen Berufsmythos der „Objektivität" eine nur schwer zu erschütternde Rechtfertigung erfährt, läßt sich die Vorgabe falscher Interessen und Ziele, falls sie getroffen wird, bestenfalls an der Diskrepanz zwischen Bekenntnissen und journalistischen "Taten " aufdecken. Höchst problematisch wird es beim Passus ,wissentlich zum Schaden der Betroffenen "; denn man muß nicht nur ein hohes Reflexionsvermögen und ein umfassendes Wissen um das, was wem ggf. nützt oder schadet, beim Kommunikator voraussetzen, sondern auch seine detaillierte Kenntnis der Rezipientenschaft und die Möglichkeit einer exakten Bezeichnung derjenigen, die im speziellen Anwendungsfall „betroffen" sind. Geht es im übrigen um soziale Konflikte und politische Auseinandersetzungen, dann wird jeder das Recht für sich in Anspruch nehmen, zumindest dem Gegner zu schaden, wo er kann. Schließlich ist auch das Unterschlagen und Verfälschen von Informationen ein weit heißeres Eisen für den „Untersuchungsrichter“, als es zunächst scheinen mag; denn: was aus dem Ereignisuniversum ist so wichtig für jeden Einzelnen innerhalb eines Massenpublikums, daß mit Sicherheit von „Unterschlagung " gesprochen werden kann, wenn es im Medium nicht berücksichtigt wird? Und wo liegen die Grenzen zwischen der notwendigen mediengerechten Gestaltung einer Information und ihrer Verfälschung? Je länger man über eine solche Arbeitshypothese diskutiert, um so unschärfer und unbrauchbarer kommt sie einem vor, und vielleicht offenbart diese Tendenz überhaupt das "Schicksal“ des Manipulationsbegriffes, der immer auf das 
allgemeine Problem objektiv ungesicherter normativer Urteile verweist. Im obengenannten Beispiel jedenfalls kommt man über die Ubung des alten Rechtsprinzips ,in dubio pro reo" kaum hinaus, es sei denn, man hat einen ganz offensichtlichen Fall einschließlich ausreichender Zugeständnisse des Kommunikators vorliegen. Hinzu kommt, daß niemand allein auch nur rein zeitlich in der Lage ist, alle öffentliche Kommunikation quasi-juristisch zu examinieren. Nur umfassende und demokratisch legitimierte Kontrollsysteme auf allen Ebenen können deshalb das Risiko, durch Manipulation publizistischer Aussagen „selbst manipuliert zu werden“, erfolgreich reduzieren; denn ohne sie ist der Einzelne den „Medien“ gegenüber weitgehend auf übermäßiges Vertrauen angewiesen. ${ }^{9}$

7. $\mathrm{Zu}$ den aktuellen strukturellen Veränderungen in der Massenkommunikation gehört die fortschreitende Pressekonzentration, und diese bietet nicht unbeträchtliche Ansatzpunkte, um die Gefahr einer wachsenden Manipulation des Einzelnen durch die Medien zu beschwören. Auch hierbei wird von den Auguren fast stets und unmittelbar wieder jene noch nicht hinreichend geprüfte Wirkungskomponente miteinbezogen, und zwar zumeist zum Nachteil der sich in der Regel aufgrund wirtschaftlicher Existenzsorgen zusammenschließenden oder kapitulierenden Verleger. Nur wenige der zahlreichen Unterstellungen in diesem Zusammenhang sind inzwischen durch empirisch-analytische Forschungsbemühungen überprüft worden, etwa betreffend die mutmaßlichen Folgen der zunehmenden Bildung lokaler Zeitungsmonopole auf Presseinhalte und politische Beeinflussungsversuche, und siehe da, die Ergebnisse sind nicht dazu angetan, alle pessimistischen Prognosen zu bestätigen. ${ }^{10}$ Das Argument der ständig geringer werdenden Alternativen für den Rezipienten durch zunehmende lokale Pressemonopole, nach dem dieser bald kaum noch echte Wahlmöglichkeiten $z$ wischen politisch unterschiedlich akzentuierenden Blättern habe, ist zwar nach wie vor recht einsichtig, doch der realistische Beobachter wird es nicht überschätzen; denn erstens war die Bandbreite politischer Akzentuierung unter den privaten Zeitungsverlagen eben noch nie breiter als die des jeweiligen gesellschaftlichen Systems, zweitens kann es sich heute tatsächlich nur noch um unterschiedliche "Akzente" handeln, da längst alle Zeitungsverlage ihr Rohmaterial zum überwiegenden Teil von denselben ganz wenigen und längst monopolisierten Agenturen beziehen, drittens ist seit langem nachgewiesen, daß der weitaus größte Teil der Rezipienten keinen Gebrauch von derartigen lokalen Wahlmöglichkeiten macht (man liest sein Blatt), viertens gibt es überregionale Blätter (wenn auch ohne Lokalteil) und fünftens Rundfunk- und Fernsehanstalten, die sich zunehmend um den Ausbau lokaler Netze und Programme bemühen.

8. Doch zurück zum Manipulationsbegriff. Was etwa bei Dröge ${ }^{11}$ im Rahmen einer marxistischen Gesellschaftstheorie implizit unterstellt wird, müßte die Wirkungsforschung erst beweisen, nämlich die tatsächliche Beeinflussung von Menschen durch bestimmte inhaltliche und formale Strategien der massenmedialen Produktion in der jeweils erwünschten Richtung.

Noelle-Neumann meint, wer den Einzelnen vor Manipulationen im Sinne von nunfreiwilligen, unbemerkten Beeinflussungen "12 bewahren wolle, müsse dafür sorgen, daß gedruckte Medien wie Zeitungen wieder an Bedeutung gewinnen; denn aufgrund ihrer "Disponibilität" (was meint, daß der Leser sich ihrer "hochselektiv" bedienen könne) seien Presseorgane die am besten geeigneten Medien zur selbstbestimmten Auswahl der angebotenen Stoffe durch den Leser, der sich nur durch diese Auswahl vor Manipulation schützen könne. Dieser Optimismus erweist sich als Idealismus, wenn 
man sich die hier und heute gegebenen subjektiven Wahlmöglichkeiten des durchschnittlichen Lesers vergegenwärtigt.

Vor dem Hintergrund des jeweiligen soziokulturellen Milieus eines Menschen, seiner Sozialisationsbedingungen und -ergebnisse sowie der konkreten jeweiligen Lesesituation redet sich nicht mehr so leicht über Wunschvorstellungen wie „Selbstschutz qua Selektionsvermögen"; denn die Mitglieder einer "pluralistischen" Gesellschaft sind unterschiedlich anfällig für Manipulationsversuche ${ }^{13}$, und es erscheint $z$.Z. wichtiger, z. B. die Ursachen für eine „hohe Anfälligkeit" aufzuspüren und zu beseitigen, als Argumente für den Bestand eines bestimmten Kommunikationsmediums zu sammeln.

9. Hier stoßen wir auf die Bedeutung der primären und sekundären Sozialisation für die Chancen bzw. Gefahren von Manipulationsversuchen. Ein aufgeklärter, fremdund selbstkritischer und gleichzeitig selbstsicherer Mensch ist zunächst der beste individuelle Schutz vor Manipulationsversuchen seitens der Massenmedien, die - selbst Institutionen der sekundären Sozialisation - so zwar nicht an der manipulativen Behandlung von Ereignissen und Inhalten gehindert werden können, denen aber doch weitgehend die Möglichkeit einer manipulativen Beeinflussung jener Menschen genommen ist, die sich z. B. der wesentlichen Widersprüche ihrer Gesellschaft stets bewußt sind. Deshalb mein erstes Plädoyer für ein integratives Gesamtkonzept von Erziehungs- und Kommunikationswissenschaftlern für Lehrer und Publizisten und die dringende Empfehlung, Massenkommunikation als Unterrichtsgegenstand und Kommunikationslehre als Unterrichtsfach in der Schule zu realisieren. ${ }^{14}$

Nur der, der einen fundierten Einblick in die ökonomischen und kommunikativen Strukturen und Prozesse im Medienbereich und darüberhinaus gewonnen hat, hat die Chance, sich zumindest eindeutigen manipulativen Inhalten und Techniken zu entziehen, weil er sie zu durchschauen gelernt hat. $\mathrm{Zu}$ einem solchen Konzept aber, und das muß ganz klar gesehen werden, gehört ein wohlbegründetes gesellschaftspolitisches Engagement; denn Manipulation ist ein strukturelles Ubel, das allen äußerst ernstzunehmenden Hindernissen und Gegnern zum Trotz endlich an der Wurzel zu packen ist, um es, soweit immer möglich, zu beseitigen.

So unvermeidlich und grundlegend menschliches Vertrauen als „Kommunikationsmedium" - auch ist, so riskant ist es angesichts mangelnder Uberprüfungs- und damit mangelnder Sanktions- bzw. Legitimationsmöglichkeiten. Im Interesse der Beseitigung sozialer Übel wie publizistischer Manipulation ist deshalb eine bessere Kontrolle der Massenkommunikation unbedingt erforderlich, ggf. durch eigens und demokratisch dafür bestimmte politische Gremien, die ihrerseits das in sie gesetzte Vertrauen einer Offentlichkeit rechtfertigen müssen, in der sie ihre eigene, sie selbst kontrollierende letzte Instanz $\mathrm{zu}$ sehen haben. ${ }^{15} \mathrm{Zu}$ den zentralen Zielen solcher Kontrollsysteme gehört, daß über die Medien für die Rezipienten endlich Wissensund Handlungsalternativen bereitgestellt werden, die wirkliche "Lebenshilfen" vermitteln (z. B. durch Verdeutlichung gesellschaftlicher Zusammenhänge vor politischen Wahlen), daß also die Welt und ihre Ereignisse in den Medien zwar noch notwendig selektiv, aber nicht mehr ohne erkennbaren Leitfaden gestückelt behandelt und verabreicht werden. Enzensberger meint in seiner bereits kritisierten Begrifflichkeit, es sei nicht die Frage, $o b$ die Medien manipuliert werden oder nicht, sondern wer sie manipuliert. ${ }^{16}$ Ersetzen wir hier "manipuliert" durch "kontrolliert", dann treffen wir genau ins Schwarze des oben skizzierten kommunikationspolitischen Ansatzes. 
10. Unter einem solchen Aspekt stellten sich nun jene oft beschworenen Gefahren technischer Manipulation und ökonomischer Konzentration in der Publizistik ganz anders dar als gewohnt. „Nicht die Technik manipuliert die Bürger", schreibt Lohmar, „sondern allenfalls deren Verwertung ohne demokratisierte Kommunikationsformen und ohne einen inhaltlich klaren gesellschaftlichen Kontext. ${ }^{{ }^{417}}$ Entsprechend darf gesagt werden, daß das eigentliche Problem der wissenschaftlichen Medienpolitik heute nicht mehr die ökonomische Konzentration sein kann, sondern daß die konsequente demokratische Kontrolle ${ }^{18}$ der Massenkommunikation das zentrale Anliegen aller sein muß, die es mit dem demokratischen Prinzip ernst meinen, wobei die Abschaffung des Privatbesitzes an einem Teil dieser Medien nach meiner Auffassung eine zwar notwendige, aber keine hinreichende Bedingung ist. Eine Demokratisierung, die sich nur auf die Verteilung und Verfügung von Produktionsmitteln und materiellen Gütern erstreckt, wird aus den oben erwähnten Gründen mangelnder "kommunikativer Kompetenz", die in den Sozialisationsbedingungen der meisten Individuen wurzelt, ebensowenig das Gespenst der Manipulation bannen können wie ein rein formal errichtetes "demokratisches" Kontrollsystem. Beide Strategien werden erst dann demokratisch sinnvoll und effektiv, wenn sie im engen Zusammenhang mit umfassenden und die Probleme der Massenkommunikation miteinbeziehenden Erziehungs- und Bildungssystemen entwickelt und verfolgt werden. Keine Frage: so etwas zu verwirklichen braucht Zeit, wahrscheinlich Generationen, so etwas muß historisch (forciert!) "wachsen ", doch kann es heute schon als Modell für eine praxisorientierte kommunikationspolitische Gesamtkonzeption fruchtbar gemacht werden.

\section{Anmerkungen:}

1. Der Aufsatz geht - redaktionell überarbeitet und punktuell weiterentwickelt - auf einen Vortrag zurüdk, den idh im November 1972 unter dem Titel „Wirkungen der Massenkommunikation: II. Manipulation" vor angehenden Realschullehrern in Lingen (Ems) gehalten habe. Nur das erste Kapitel und einige Passagen im vorletzten habe ich dem bei gleicher Gelegenheit gehaltenen Referat ,Wirkungen der Massenkommunikation: I. Sozialisation" übernommen, das im übrigen aus einer Darstellung bekannter kommunikationswissenschaftlicher Thesen und Resultate besteht und zu dessen Gegenstand längst einige wesentlid gründlichere Darstellungen vorliegen. Verwiesen sei vor allem auf Frank Bledjian / Christa Stosberg: Analyse der Massenkommunikation: Wirkungen. Düsseldorf 1972; Kurt Koszyk: Wirkungen der Massenkommunikation. Ergebnisse und Kritik einer Forschungsrichtung. In: Aus Politik und Zeitgeschichte. Beilage zur Wochenzeitung „Das Parlament". B 39 (23. September) 1972, S. 3-45; und Franz Ronneberger: Sozialisation durch Massenkommunikation. In: Franz Ronneberger (Hrsg.): Sozialisation durch Massenkommunikation. Der Mensch als soziales und personales Wesen, Bd. IV. Stuttgart 1971, S. 32-125.

2. Vgl. zu diesem Kapitel Franz Ronneberger: a.a.O., S. $70 \mathrm{ff}$.

3. Vgl. Herbert Schnädelbach: Was ist Ideologie? In: "Das Argument" 50, Karlsruhe 1969, S. $83 \mathrm{f}$.

4. So meint Holzer, daß die Grundlagen der Manipulation in der Verpflichtung der Massenmedien auf das herrschende kapitalistische System liegen: "Aufgrund ihrer politökonomischen Lage und der Entfremdungssituation des Publikums müssen die Medien jene Techniken anwenden, die das Denken der Mehrheit der Bevölkerung so regulieren, daß es den profit- und wahlstimmenorientierten Interessen der Machtgruppen in Wirtschaft und Politik entspridit. " Vgl. Horst Holzer: Politik in Massenmedien Zum Antagonismus von Presse- und Gewerbefreiheit. In: Ralf Zoll (Hrsg.): Manipulation der Meinungsbildung. Opladen 1971, S. 76.

5. Vgl. Johannes Gross: Zu viel oder zu wenig Offentlichkeit? Neue Mittel der Manipulation. In: „Frankfurter Allgemeine Zeitung“ v. 3.11.1972. 
6. So gesehen klingt z. B. der Manipulationsvorwurf der politischen Linken merkwürdig moralistisch, wiewohl er auf der richtigen Einsicht beruht, daß sich fast alle publizistischen Produktionsmittel in der BRD fest in der Hand des "politischen Gegners" befinden. Enzensberger setzt sich energisch von diesem normativen Gebrauch des Manipulationsbegriffes $a b$, den er für defensiv und defätistisch hält, und definiert: „Manipulation, zu deutsch Hand- oder Kunstgriff, heißt soviel wie zielbewußtes technisches Eingreifen in ein gegebenes Material. Wenn es sich um ein gesellschaftlich unmittelbar relevantes Eingreifen handelt, ist die Manipulation ein politischer Akt. Das ist in der BewußtseinsIndustrie prinzipiell der Fall.“ Vgl. Hans Magnus Enzensberger: Baukasten zu einer Theorie der Medien. In: „Kursbuch“ 20 (März 1970), S. 166.

7. Diese Arbeitsdefinition wurde im Rahmen eines Aufbaukurses für Volontäre und Jungredakteure entwickelt, den das Deutsche Institut für Publizistische Bildungsarbeit vom 23. bis 31. Mai 1972 in Düsseldorf-Hassels veranstaltete. Sie findet sich im "Arbeitsbericht Gruppe I. Kommunikation".

8. Zum neuesten Stand der Wirkungsforschung vgl. Elisabeth Noelle-Neumann: Kumulation, Konsonanz und Offentlichkeitseffekt. In: „Publizistik“, 18:1973, 26-55.

9. Näheres zu diesem Argument in meinem „Entwurf einer kommunikationspolitischen Gesamtkonzeption“, der zu Beginn des SS 1973 anläßlich des Seminars „Kommunikationspolitik“ am Institut für Publizistik in Münster als Manuskript gedruckt erschienen ist.

10. Vgl. Manfred Knoche / Winfried Schulz: Folgen des Lokalmonopols von Tageszeitungen. Eine vergleichende Inhaltsanalyse des Lokalteils von Monopol- und Wettbewerbszeitungen. In: „Publizistik“, 14:1969, 298-310, sowie neuerdings die Untersuchung der „Folgen lokaler Zeitungsmonopole ${ }^{\alpha}$ von Elisabeth Noelle-Neumann und Klaus Schönbach, deren Ergebnisse u. a. referiert wurden in: „Der Journalist“, Nr. 6/1974, S. 22-26: E.NoelleNeumann: Folgen lokaler Zeitungsmonopole.

11. Dröge spricht mit Einschränkung auf die Massenpresse von thematischer und normativer Standardisierung und Homogenisierung, von manifester Minderheitendiskriminierung, von Unterstützung und Stabilisierung der etablierten Interessen- und Wissensträger, also der herrschenden gesellschaftlichen Individuen und Gruppen, von „entpolitisierenden“ Gestaltungsprinzipien wie der Personalisierung sozialer und politischer Tatbestände, der Privatisierung öffentlicher Belange, der Mystifizierung von Politik durch die Konstruktion sogenannter Schicksalsideologien, der Detaillierung von Zusammenhängen in unverbundene Informationspartikel und der Generalisierung, d.h. der Beschwörung von Zusammenhängen ohne Berücksichtigung ihrer strukturellen Bedingungen. Er räumt dieser Presse einen bedeutenden Anteil an der gesellschaftlichen Ideologiebildung und -bewahrung ein (Ideologie im marxistischen Sinne); denn ihre drei wesentlichen Funktionen seien a) die Verschleierung sozialer Widersprüche, b) die Kompensation von individuellen Opfern und Frustrationen durch psychische Ersatzbefriedigungsmechanismen, und c) die permanente Rationalisierung und Legitimation der bestehenden Verhältnisse. Vgl. Franz Dröge: Einführung in die Medienanalyse der BRD. Münster 1972 (als Manuskript gedrucktes Vorlesungsskript), S. 65 f. und S. $91 \mathrm{ff}$.

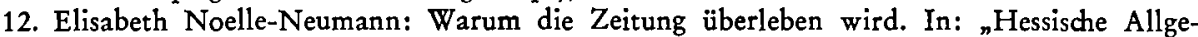
meine ${ }^{\alpha}$, Kassel, v. 5. 10. 1972.

13. Schmidtchen z. B. nennt fünf psychische Faktoren, die den Einzelnen mehr oder weniger manipulationsgefährdet machen: Angst, Vorurteile (Projektions- bzw. Abwehrmechanismen), geringe Selbstachtung, kognitive Dissonanz und die Tendenz, soziale Sanktionen zu vermeiden ("Mitläufereffekt ${ }^{\circ}$ ). Vgl. Gerhardt Schmidtchen: Manipulation durch die Massenmedien. In: Gerhardt Schmidtchen: Manipulation - Freiheit negativ. Berlin 1970, S. $27 \mathrm{ff}$.

14. Hiermit stelle ich mich hinter die Forderungen des Autorenkollektivs Robert Krauthausen, Gert Schukies, Franz R. Stuke und Dieter Warstat: „Kommunikationslehre“ statt ${ }_{n}$ Medienerziehung ${ }^{\alpha}$. In: „Aula ${ }^{\alpha}$, Heft 1 (Februar) 1972.

15. Vgl. meinen oben zitierten „Entwurf ... .

16. Vgl. Hans Magnus Enzensberger: a.a.O., S. 166.

17. Ulrich Lohmar: Die Produktivkraft Wissenschaft als publizistisches Problem. In: Aus Politik und Zeitgeschichte. Beilage zur Wochenzeitung "Das Parlament". B 21/72 (20. Mai 1972), S. 13.

18. Kritisch zur Ausweitung von "Kontrolle ${ }^{\text {a }}$ äßert sich in diesem Heft von CS M. Schmolke: Informierte Welt - bessere Welt? Vgl. S. 301-311. 


\section{S U M M A R Y}

The effects of massmedia are constantly under discussion. Not only good or bad effects are realized today, but there are also talks about manipulation through mass media. But is there a society which is in any way free from any manipulation? Do not, for example, marketoriented social systems promote the trend to manipulation monopolies? The author thinks that not the concentration but the democratic control of mass communication must be the central aim. Thus he promotes a general communications concept for society.

\section{RÉSUMÉ}

Les effets des mass media sont constamment sujet à discussion. De nos jours, on ne parle plus seulement de bonnes ou de mauvaises influences, mais de "manipulation" des êtres humains par les mass media. Mais, existe-t-il une société libre de toute manipulation? Et les systèmes sociaux orientés vers une économie de marché n'encouragent-ils pas l'aspiration des groupes possédant l'autorité vers des "monopoles de manipulation"? L'auteur tombe sur la notion de manipulation et en vient à la conclusion suivante: pas la concentration, mais le contrôle démocratique des mass media doit être le désir de tous. Il prend fait et cause pour le développement d'une conception totale de politique de communication appropriée.

\section{RESUMEN}

Las repercusiones de los medios de comunicación social son objeto permanente de discursión. Hoy se habla no solo de efectos buenos y perniciosos sino de "manipulación" del hombre a través de los medios de comunicación. Pero es que existe una sociedad libre de manipulación? Acaso no favorecen los sistemas sociales orientados al libre mercado la tendencia de los grupos poderosos al monopolio de la manipulación"? El autor arremete contra el concepto Manipulación y llega a la conclusión de que no la concentración sino el control democrático de la comunicación de masas debe ser deseo central de todos. Es partidario del desarrollo de una adecuada concepción global político-comunitaria. 